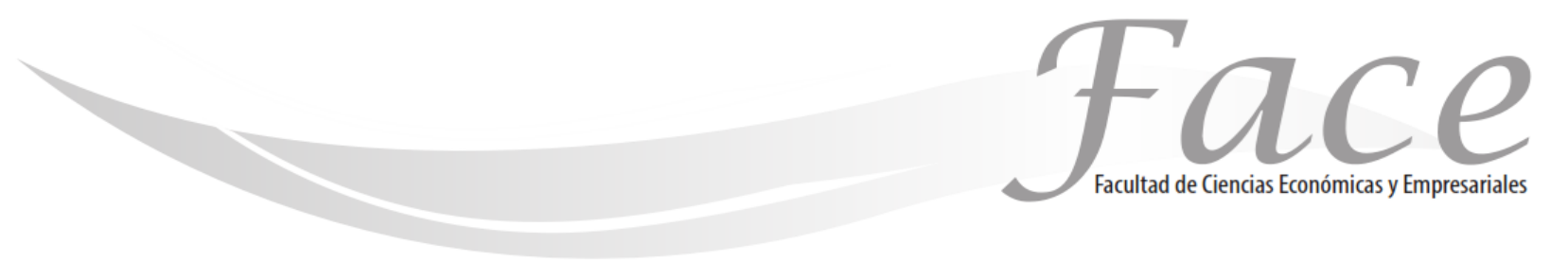

ISSN Impreso: 1794-9920 ISSN Electrónico: 2500-9338

Volumen $16-\mathrm{N}^{\circ} 1$

Año 2016

Págs. 79 - 89

\title{
LA GESTIÓN DEL CICLO DE VIDA DE PROYECTOS EN PEQUEÑAS ORGANIZACIONES COMUNITARIAS: UNA PROPUESTA METODOLÓGICA
}

\author{
Meynsser Liliana Montero Acosta * \\ Enlace ORCID: http://orcid.org/0000-0003-1977-8595
}

Fecha de Recepción: 5 de Julio 2016

Fecha de Aprobación: 30 de Octubre 2016

\section{Resumen:}

En este trabajo de investigación, se propone una metodología para la gestión del ciclo de vida de proyectos, la cual ha sido adaptada a las problemáticas de los pequeños productores del sector panelero de Villeta - Cundinamarca en Colombia. Para conseguir el propósito de la investigación se aplicó enfoque cualitativo, con una combinación de dos modalidades: análisis documental, para la Fase I e investigación-acción para la Fase II.

Como resultado del proceso de investigación, se presenta la estructura metodológica, así como la estrategia de implementación en el contexto bajo estudio, y la necesidad de desarrollar capacidades para una adecuada gestión de los proyectos. Los principales hallazgos consideran que utilizar metodologías de proyectos que se desarrollen con la participación de los productores desde su inicio y a lo largo del ciclo de vida de los mismos, influirá en resultados ajustados a sus necesidades y expectativas.

Palabras Claves: Estructuración de Proyectos, PYMES, Colombia, producción panelera.

*Investigadora. Departamento de Proyectos, facultad de Ingenierías. Universidad EAN - Colombia. Contacto: mmontero_2@universidadean.edu.co. 


\title{
THE MANAGEMENT OF THE LIFE CYCLE OF PROJECTS IN SMALL COMMUNITY ORGANIZATIONS: A METHODOLOGICAL PROPOSAL
}

\begin{abstract}
:
In this research, we propose a methodology for project life cycle management, which has been adapted to the problems of the small producers of the panel sector of Villeta - Cundinamarca in Colombia. To achieve the purpose of the research, a qualitative approach was applied, with a combination of two modalities: documentary analysis for Phase I and action research for Phase II.

As a result of the research process, it presents the methodological structure, as well as the implementation strategy in the context under study, and the need to develop capacities for an adequate management of the projects. The main findings consider that using project methodologies that are developed with the participation of producers from the beginning and throughout their life cycle, will influence results adjusted to their needs and expectations.
\end{abstract}

Keywords: Structuring of Projects, SMEs, Colombia, panela production.

\section{A GESTÃO DO CICLO DE VIDA DOS PROJECTOS EM PEQUENAS ORGANIZAÇÕES COMUNITÁRIAS: UMA PROPOSTA METODOLÓGICA}

\begin{abstract}
Resumo:
Nesta pesquisa, propomos uma metodologia para o gerenciamento do ciclo de vida do projeto, que foi adaptada aos problemas dos pequenos produtores do setor de painel de Villeta - Cundinamarca na Colômbia. Para atingir o objetivo da pesquisa, foi aplicada uma abordagem qualitativa, com uma combinação de duas modalidades: análise documental para a Fase I e pesquisaação para a Fase II.

Como resultado do processo de pesquisa, apresenta a estrutura metodológica, bem como a estratégia de implementação no contexto em estudo, ea necessidade de desenvolver capacidades para uma adequada gestão dos projetos. Os principais achados consideram que o uso de metodologias de projeto desenvolvidas com a participação dos produtores desde o início e ao longo de seu ciclo de vida, influenciará os resultados ajustados às suas necessidades e expectativas.
\end{abstract}

Palavras-Chave: Estruturação de Projetos, PME, Colômbia, produção panela. 


\section{INTRODUCCIÓN:}

Meynsser Liliana Montero Acosta

Uno de los grandes aportes que se han materializado con el desarrollo del área de conocimiento de la Dirección de Proyectos, es precisamente la incorporación de procesos sistemáticos de gestión para organizar todo el ciclo de vida de los proyectos en muchos sectores. En esa dinámica, un factor clave dentro de dicho proceso, consiste en utilizar buenas prácticas que se aplican desde la identificación de proyectos adecuados, es decir que respondan realmente a una problemática o una expectativa real de desarrollo de un sector, hasta su puesta en marcha de forma exitosa y sostenible.

En ese sentido, la adaptación de nuevas aproximaciones metodológicas que ayuden a gestionar nuevas iniciativas de desarrollo con un enfoque de proyectos, además de fortalecer las capacidades de los ciudadanos, para realizar cada vez más y mejores proyectos, es una necesidad latente en muchas regiones y para muchas actividades productivas tradicionales del contexto colombiano. No es un secreto que, para Colombia, en todos los sectores y actividades, en términos de capacidades de identificación, formulación, estructuración, y ejecución de proyectos, aún hay mucho camino por avanzar y mejorar.

Una de esas actividades productivas tradicionales de Colombia, donde se pueden generar muchos beneficios a partir de la estructuración de más y mejores proyectos, es el cultivo de la caña panelera, debido a su importancia en varias zonas del territorio nacional, tanto en su uso de mano de obra, como en la contribución al Producto Interno Bruto Agropecuario. El país panelero está en un proceso de modernización, impulsado desde muchos frentes, algunos estatales, otros en su gran mayoría de mercado, generando un panorama diferente para aquellos vinculados con la producción de panela (Gómez Maluchi FEDEPANELA - 2009), promoviendo con ello la necesidad de nuevos proyectos que aporten a dicha modernización, mejorando con ello la calidad de vida de todos los involucrados de la cadena productiva.

Dentro de las regiones productoras de panela del país, se encuentra el municipio de Villeta - Cundinamarca, caracterizado por pequeños y medianos productores, y cuyo caso específico ha sido objeto de estudio en este trabajo de investigación. En este contexto geográfico se ha podido apreciar que los pequeños productores de panela requieren modernizar y adaptar sus sistemas productivos de acuerdo a las exigencias y normativas gubernamentales, para que puedan ser competitivos frente a la globalización de la economía y la apertura de nuevos mercados. Sin embargo, debido a la baja posibilidad de autofinanciarse para lograrlo, se convierten en problemáticas que, a pesar de los esfuerzos realizados por entes gubernamentales y privados, es necesario trabajar desde la misma comunidad y para la comunidad, lo cual tiene un punto en común, que tengan la capacidad de autoidentificar y auto-estructurar los proyectos que requieren.

Con el propósito de brindar un aporte a la solución de estas problemáticas, se inició ésta investigación en junio de 2015, utilizando un enfoque cualitativo, dirigido en dos fases, la primera: evaluar, diagnosticar y proponer una metodología del Ciclo de Vida de Proyectos adaptada a las problemáticas del sector panelero colombiano. Dicha metodología muestra las diferentes etapas que recorre un proyecto desde que se concibe la idea hasta que se materializa en una acción concreta, adaptadas al entorno del sector, estas etapas son: "diagnóstico", "factibilidad", "inversión", "operación" y "evaluación ex-post". La primera fase de la investigación se realizó a través de un análisis documental, revisando, recopilando y analizando fuentes tanto de gestión de proyectos como de las principales situaciones vividas por los pequeños productores de Villeta.

Para la segunda fase, utilizando la modalidad de investigación-acción, que requiere la participación de los posibles beneficiarios de las soluciones que pueden surgir de la aplicación de la metodología propuesta, se plantea realizar una evaluación de las capacidades actuales de la región en cuanto a gerencia de proyectos, estableciendo con ello un portafolio de proyectos basado en los criterios establecidos con la comunidad, y a partir de la implementación de la metodología en proyectos piloto fortalecer también las capacidades en gerencia de proyectos ajustadas a las necesidades del sector.

Con la metodología propuesta se pretende que los pequeños productores se conviertan en los actores principales en la comprobación del modelo construido, y a la vez evaluar su efectividad sobre la realidad que vive actualmente su región. Significa entonces que, a través de los instrumentos y herramientas que proporciona la metodología, se busca potencializar el conocimiento y experiencia de los productores para ser usado en beneficio propio, de sus familias y de su comunidad.

\section{MARCO TEÓRICO:}

\subsection{Metodologías y estándares del ciclo de vida de proyectos}

Uno de los enfoques de este trabajo toma línea en el desarrollo de una metodología del ciclo de vida de 
proyectos para pequeñas organizaciones comunitarias, por lo tanto, para la aproximación teórica es necesario entender algunos conceptos sobre este tipo de metodologías, los proyectos de desarrollo y algunas de sus aplicaciones en general, los cuáles son desarrollados a continuación. Se ha buscado, en cada caso, encontrar la relación y aporte que pueda presentar entre el cuerpo teórico y el propósito del trabajo de investigación, para con ello nutrir la propuesta metodológica presentada.

Para empezar, y como su nombre lo indica, los proyectos o programas de desarrollo tienen como finalidad principal obtener resultados que permitan el crecimiento social y económico de las regiones o los países que los realicen. Adicionalmente al cumplimiento de la triple restricción (alcance, costo y tiempo) que aplica para proyectos 0 programas en general, para los proyectos de desarrollo se toma como punto de partida unos supuestos de carácter social y de capital que generarán un cambio progresivo visible a través de la entrega de resultados intermedios que finalmente ocasionan una mejora sostenible en el tiempo.

Ahora bien, con respecto a intervenciones en la realidad para alcanzar cambios positivos, Ortíz y Rivero (2007) expresan: "Intervenciones de alto impacto, costo efectivas, y sostenibles requieren, en principio, un claro entendimiento del conjunto de los elementos, las relaciones, y dinámicas que existen dentro de una determinada realidad. En la medida que exista una comprensión sólida, se podrán formular, validar, implementar, y evaluar de manera más consistente las diversas acciones asociadas a la gestión de un proyecto y/o programa en sus diversos momentos (identificación de necesidades, definición de intervenciones, selección de alternativas, asignación de recursos, implementación de la estrategia, y evaluación y aprendizaje)".

Por otro lado en todo proyecto o programa de desarrollo, su planificación es vital para lograr el éxito del mismo. Una mala planificación o una ausencia de ella, puede dar como resultado un fracaso del proyecto o programa. A lo largo del tiempo se ha desarrollado diferentes herramientas para contribuir a una buena y acertada planificación de un proyecto o programa, que logre cumplir con el propósito para el cual se creó y dentro del tiempo y el costo estimado.

Es así como en muchas ocasiones se puede encontrar, dentro de la planificación de los proyectos, una buena estimación en cuanto a los requerimientos materiales, financieros, de personal, de tiempo y de los entregables esperados. Pero, a menudo no se tienen en cuenta factores fundamentales como son: el correcto análisis de los objetivos, de los grupos de interés y de los factores externos que llegan a propiciar el éxito o el fracaso del proyecto o programa.
Adicional a las falencias de la planificación, los sistemas de seguimiento son insuficientes y poco adecuados, centrándose en la consecución de los entregables físicos y dejando de lado las condiciones y las estrategias del proyecto o programa.

Todo esto trae como consecuencia resultados inesperados y no respondan a las necesidades de los grupos de interés. Los proyectos o programas pueden obtener resultados adversos imprevistos que seguramente pueden evitarse con una planificación más metódica.

Es por esto que, en repetidas ocasiones las organizaciones patrocinadoras se han propuesto mejorar la estructuración y la planificación de los proyectos o programas; al igual que enfatizar en el análisis y la evaluación a lo largo del ciclo de vida de los proyectos o programas. Muchos resultados a lo largo de la historia han demostrado que una mejor y mayor planificación redunda en proyectos más exitosos y con ahorros evidentes. En numerosos casos las ventajas y resultados pueden ser significativos y valiosos para la comunidad.

Un ejemplo de ello es el enfoque del Marco Lógico (EML) que responde precisamente a muchos de los problemas descritos anteriormente, proporcionando los lineamientos con respecto a la planificación y la gestión de proyectos 0 programas de desarrollo (ILPES, 2004). El método del "Marco Lógico" es una manera de organizar los principales elementos de un proyecto o programa, destacando las relaciones existentes entre los costos estimados, la planificación de las actividades y el beneficio esperado.

IPLES (2005), manifiesta que aunque el Marco Lógico es mundialmente reconocido como una herramienta que tiene como propósito la planificación, ejecución y evaluación de los proyectos de desarrollo, su estructura y desarrollo no se enfoca en la planificación sino en la evaluación, es decir que procuran responden las siguientes preguntas:

- ¿El proyecto o programa proporcionó el resultado esperado?

- ¿Con éste proyecto o programa se obtuvo el desempeño deseado?

- ¿Se logró el impacto adecuado a través de los medios y los recursos utilizados?

- Si el resultado no se dio. ¿Qué fue lo que pasó y por qué?

Dicho de otra manera, determinar cuáles son las causas y los porqué que originaron los inconvenientes del proyecto 0 programa evaluado.

Pero, fue a finales de la década de los 60 , cuando los esfuerzos se enfocaban hacia una mejora significativa en los procedimientos y metodologías de evaluación, que se 
Meynsser Liliana Montero Acosta

propició una nueva e innovadora idea del proceso de evaluación y de sus herramientas. La Unidad de evaluación de la Agencia para el Desarrollo Internacional de Estados Unidos de América (USAID) toma la iniciativa para la definición de una nueva metodología de evaluación mejorada (Aldunate y Córdoba, 2011).

En tal sentido ésta propuesta buscaba responder a la necesidad de mejora de los sistemas de evaluación, pero Fry \& Associates en su primer informe manifiesta que el principal inconveniente realmente radicaba en la estructuración del proyecto:

"The real obstacle to good project evaluation was the absence of clearly defined program and project objectives and targets by which to measure them. In this report were the essential first elements of the logical framework, its dialectic, and its linking of project design to sound evaluation"

De acuerdo con éste razonamiento, Fry \& Associates manifestaba claramente que la ausencia de elementos que permitieran medir el proyecto o programa, era el mayor inconveniente presentado en los proyectos y programas. Elementos tales como definición del programa, objetivos del proyecto, metas a alcanzar y resultados esperados, darían una estructuración adecuada que adicional facilitarían la formalización y asignación de responsabilidades y a su vez serian la base para una adecuada evaluación de los proyectos o programas de desarrollo.

Tiempo después y luego de estudiar las recomendaciones de Fry \& Associates, USAID contrató para ésta segunda parte del proceso a Practical Concepts Inc., abordando el diseño, desarrollo y la aplicación de una visión de Marco Lógico (Logical Framework Approach) en las actividades y proyectos al interior de USAID. Esta segunda fase se realizó durante 1969 y 1970.

Por ello, el Marco Lógico (ML) desarrollado por USAID tenía en cuenta tres inconvenientes que se presentaban en los proyectos de desarrollo:

- Ambigüedades en la Planificación. La planificación de estos carecía de precisión, con objetivos múltiples, que no estaban claramente relacionados con las actividades del proyecto.

- Responsabilidad gerencial imprecisa. Los proyectos no se ejecutaban con éxito, y no había distinción entre los factores de éxito a cargo del gerente del proyecto, de los factores fuera de su control.

- Evaluación poco imparcial. No había una herramienta de medición de los logros del proyecto y los evaluadores no tenían una base clara y objetiva para hacer seguimiento y evaluación de los resultados obtenidos con el proyecto.
Adicional a que el enfoque del Marco Lógico supera estos problemas, también ofrece unas ventajas adicionales, como por ejemplo:

- Pensar en forma coherente y estructurada.

- Facilitar los acuerdos entre los actores acerca de los logros del proyecto.

- Permitir un enfoque técnico de los aspectos críticos del proyecto.

- Acortar documentos de proyecto que de otras maneras se hacen extensos y poco concretos.

- Suministrar información clave para la ejecución, seguimiento y evaluación del proyecto.

Luego de los primeros acercamientos sobre el método, la primera versión propiamente dicha de "Marco Lógico" fue materializada por Rosemberg y Posner en el año de 1979. Durante la década de los 70, esta versión sirve como base para desarrollos perfeccionados con nuevos aportes y mejoras hasta converger en las actuales formulaciones metodológicas, entre las cuales cabe destacar la adaptación realizada por la GTZ en 1983 (de las más elaboradas) y origen de la metodología alemana ZOPP (Ziel Orientierte Projekt Planung). Otras aplicaciones basadas en el mismo, son las del Programa de las Naciones Unidas para el Desarrollo "PNUD" y el Banco Mundial y las más recientes que se enfocan en una nueva cultura Gestión para resultados como la desarrollada por la Organización de la Naciones Unidas para la alimentación y la agricultura (FAO).

De igual forma, actualmente existen varias organizaciones internacionales dedicadas a establecer estándares para la gestión de proyectos. Entre otras, PMI con PMBoK, OGC con PRINCE2, IPMA con ICB, APM con APMBoK, ISO con ISO 21500, Easel Corporation con Scrum, Toyota con Kanban (Crawford, 2004). Algunas de estas organizaciones proponen metodologías, otras pautas o esquemas que permiten a las organizaciones y a los profesionales aplicar buenas prácticas en la realización de sus proyectos. Hoy en día, la gerencia de proyectos se ha convertido en una solución de primera clase en las operaciones comerciales. Las organizaciones grandes 0 pequeñas, públicas 0 privadas, reconocen que un enfoque estructurado para la planificación y gestión de proyectos es una competencia básica necesaria para el éxito.

Como complemento de esto se tiene en cuenta el documento de Gestión Pública con base en resultados (2014) donde "Por más de diez años, la Organización de las Naciones Unidas para la Alimentación y la Agricultura (FAO), a través de proyectos continuos de evaluación y análisis de políticas rurales, ha brindado asistencia técnica a la Secretaría de Agricultura, Ganadería, Desarrollo Rural, Pesca y Alimentación (SAGARPA) de México, en materia de evaluación de programas de fomento productivo agropecuario, pesquero, acuícola y de desarrollo rural. En 
el marco de esta cooperación, se han desarrollado de manera conjunta herramientas metodológicas para la gestión de las distintas fases de un programa: diagnóstico sectorial, diseño, implementación y evaluación".

\subsection{Marco Referencial: La producción de panela en Colombia}

De acuerdo con la $\mathrm{FAO}^{1}$, la producción de la panela es una de las más tradicionales agroindustrias rurales en América Latina y el Caribe y la producción mundial de este producto se encuentra en alrededor de 13 millones de toneladas por año. En Colombia, este proceso, que inició con el cultivo de caña de azúcar, existe desde la época de la conquista, y con su introducción al país se originó la producción de panela, la cual actualmente se realiza en 25 de los 32 departamentos de Colombia, y constituye uno de los elementos esenciales de la canasta familiar de los hogares de estrato medio y bajo del país.

Desde esa época, con la caña de azúcar llegaron también los trapiches y la molienda a extensas regiones de nuestro País. Miles de familias campesinas, con un laborioso proceso completamente natural, han convertido el jugo de la caña en este producto alimenticio, de reconocidas cualidades energéticas y nutricionales.

Por otro lado, se destacan dos canales de distribución de la panela en el país: el primero corresponde a las zonas planas del Valle del Cauca y Risaralda; los productores despachan la panela directamente a los supermercados 0 a las plazas de mercado local, para su venta al consumidor. El segundo corresponde al resto de regiones paneleras del país, típicas de pequeños y medianos productores, en el que interviene una cadena más larga de agentes comercializadores (el acopiador, el mayorista y el minorista o detallista). También se presentan en esta cadena otros intermediarios y cooperativas de productores (Osorio, 2007, p. 145). En Colombia, el departamento de Cundinamarca abarca un $20,30 \%$ de la producción nacional y es allí donde se espera realizar el trabajo de campo, específicamente en la población de Villeta.

Un referente importante dentro de esta investigación es el documento de grado realizado por Luz Lilia Ubaque González (2013) Ilamado "Gestión en la producción panelera, municipio de Villeta, Cundinamarca", allí se identifica claramente y bajo la participación activa de los diferentes involucrados, las diferentes situaciones asociadas a la producción de panela de la región.

En los últimos años, instituciones como la Gobernación de Cundinamarca, a través de la Secretaría de Agricultura,

\footnotetext{
${ }^{1}$ FAO. Panela Production as a Strategy for Diversifying
} Incomes in Rural Area of Latin America. Rome. 2007.
FEDEPANELA, CORPOICA, ICA y las UMATAS han realizado esfuerzos para fortalecer el sector, presentando proyectos y utilizando principalmente la Metodología General Ajustada (MGA) para su estructuración y ejecución. Sin embargo, el sector panelero de Villeta sigue presentando dificultades en el propósito de fortalecer la producción de la panela. Es por ello que es de suma importancia avalar si los esfuerzos que se realizan tienen una respuesta beneficiosa para estos pequeños propietarios y naturalmente para la producción panelera del país. Para esto, es necesario que se propicien espacios participativos con la comunidad para que ellos aporten en la construcción de alternativas que permitan generar proyectos bien estructurados y de esta manera poder obtener recursos para su realización, motivación principal de ésta investigación.

Frente a los diferentes proyectos que se han dado en la región y como Ubaque lo describe en su documento, es necesario el fortalecimiento y consolidación de las familias paneleras a través de la participación de la comunidad en espacios colectivos para que desde allí se aporte al desarrollo efectivo del sector de manera continua y a largo plazo.

Es por esto que el resumen de Ubaque donde especifica lineamientos de gestión que contribuyan al fortalecimiento de la dinámica de adaptación a los cambios de los sistemas tradicionales de producción de panela en el municipio de Villeta - Cundinamarca, es el punto de partida para la aplicación de la metodología del ciclo de vida del proyecto al que se refiere esta investigación y con el fin de comprobar como dicha metodología puede propiciar la participación de la comunidad en la realización de proyectos que les ayuden a resolver conjuntamente las situaciones presentadas en el sector.

Lo que se pretende demostrar es si la aplicación de esta metodología logrará mejorar el desempeño y el éxito en la solución de la problemáticas del sector panelero de Villeta - Cundinamarca. Así mismo, se busca promover la participación activa de los pequeños productores en la búsqueda de las alternativas de solución de sus situaciones. Por último se espera que ésta metodología sea referente para muchos otros sectores que presenten características similares con posibilidades limitadas y que puedan a través de unir esfuerzos conseguir los resultados esperados.

\section{METODOLOGÍA:}

Este estudio comenzó en junio de 2015, una vez se tuvo claridad sobre el objeto de ésta investigación, se definieron 


\section{Meynsser Liliana Montero Acosta}

los objetivos y la pregunta a resolver, se determinó cuál sería el enfoque metodológico de la investigación con el fin de establecer la manera apropiada de hacerlo, es así como se planteó su desarrollo desde el enfoque de investigación cualitativa. Este enfoque permite realizar un análisis de la realidad social de un sector, a través de la indagación del comportamiento en una situación específica (Báez y De Tudela, 2007). Luego de elegir el enfoque, se realizó la elección de la combinación de dos modalidades de investigación cualitativa: el análisis documental y la investigación-acción aplicadas en las dos fases que componen esta investigación:

Para la primera fase se utilizó la modalidad de análisis documental apuntando a dos frentes definidos: el primer frente se enfocó en la revisión de fuentes de metodologías, guías y estándares que cubren todo o parte del ciclo de vida de proyectos utilizados a nivel mundial, así como referentes de sus aplicaciones en diferentes países y sectores económicos. Para el segundo, frente la revisión de fuentes, estuvo dirigida a estudiar la situación actual de los pequeños productores de panela del sector de Villeta Cundinamarca.

El primer frente permitió identificar, recopilar y analizar una serie de documentos relevantes para el propósito establecido, por un lado se realizó una revisión minuciosa de documentos de EI BID, la CEPAL, la CIDEAL, la FAO, el PMI, VMEdu, SGS y demás organismos que se han preocupado por establecer modelos que permitan obtener soluciones y sostenibilidad en proyectos y programas de desarrollo. Por otro lado, se analizaron los resultados obtenidos en sus aplicaciones y las técnicas utilizadas en dicha aplicación. Como referentes principales de ésta primer frente se encuentran Manual de Formulación de Proyectos de Cooperación Internaciona (Acción Social, 2006), Metodología de proyectos de cooperación para el desarrollo ( $\mathrm{AECl}, 1999)$, El enfoque del marco lógico: 10 casos prácticos (Camacho, Cámara, Cascante y Sainz, 2001), Orientaciones para la aplicación del enfoque del marco lógico - Errores frecuentes y sugerencias para evitarlos (Gómez y Cámara, 2003), El ciclo del proyecto de cooperación al desarrollo (Gómez y Sainz, 1999) y Metodología del marco lógico para la planificación, el seguimiento y la evaluación de proyectos y programas (Ortegón, Pacheco y Prieto, 2005); todas ellas aportaron para el diseño y estructuración de la metodología propuesta.

En el segundo frente, se recopilaron y analizaron documentos de organismos como FEDEPANELA, el BID, la Superintendencia de Industria y Comercio, el SENA, el ICA y documentos de investigación realizados por estudiantes de universidades, que permitieron identificar las principales situaciones vividas por los pequeños productores de panela de la región de Villeta. También permitieron realizar una comparación de estas con los resultados obtenidos en otras regiones del país donde la producción y comercialización de la panela tienen problemáticas similares 0 han obtenidos resultados satisfactorios para la región. Entre los documentos estudiados en el segundo frente se encuentran principalmente: Estudios de (Delegatura de Protección de la Competencia, 2010-2012), Formular y evaluar el proyecto de producción de panela en la vereda la Mazata del municipio de Villeta Cundinamarca (Montero, 2008) y Panela production as a strategy for diversifying incomes in rural area of Latin America (Rodriguez, García, Roa y Santacoloma, 2008), estos sirvieron como referencia para identificar la situación de los pequeños productores de panela del sector.

Para la segunda fase, se utilizará la modalidad de investigación-acción que requiere la participación de los posibles beneficiarios de las soluciones que pueden surgir de la aplicación de la metodología propuesta. De esta manera, los pequeños productores se convierten en los actores principales en la comprobación del modelo construido y su efectividad sobre la realidad que viven actualmente en su región. Como Lewin, Kurt (1946) lo propone, los dos principales componentes de la investigación-acción son la participación de las decisiones de grupo y el compromiso de la mejora del equipo. Kemmis y Mactaggart (1988) en su libro "Como planificar la investigación-acción" nombran la característica distintiva que su autor Lewin atribuye a las personas que se ven afectadas por este tipo de cambios previstos, teniendo ellos la primera responsabilidad de la decisión sobre la orientación que le darán a estas acciones que pueden conducir a la mejora.

\section{RESULTADOS Y PROPUESTA}

Para los programas y proyectos de inversión a nivel gubernamental la metodología que más se utiliza es la Metodología General Ajustada (MGA), basado en metodologías mundialmente conocidas como son el Método de Marco Lógico (MML) de origen estadounidense y la Planificación de proyectos orientada a objetivos (ZOPP) de origen alemán. Esta metodología permite a través del registro en un aplicativo, identificar y estructurar los proyectos para acceder a los recursos y posteriormente permite la evaluación de sus resultados.

Pese a que son metodologías que históricamente han sido probadas de manera exitosa, en la zona bajo estudio (Villeta) no han logrado un proceso contundente en referencia a la agroindustria panelera, esto debido a que los proyectos no se desarrollan de manera integral con la participación de los productores desde su fase inicial y a lo 
largo del ciclo de vida de los mismos. La no participación de la comunidad desde el inicio de los proyectos ha traído como consecuencia que algunos de ellos terminen en esfuerzos fallidos y no cumplan con las expectativas de los pequeños productores trayendo como consecuencia su falta de credibilidad sobre nuevas propuestas.

Con base en la información recopilada que recoge las experiencias obtenidas de los proyectos desarrollados junto con experiencias recogidas de la práctica profesional de los últimos años y con un estudio exhaustivo de los diferentes estándares para la gestión de proyectos existentes, se pudo conjugar de manera adecuada una única metodología que abarque desde la ideación pasando por el diagnóstico, factibilidad, inversión, seguimiento, operación y evaluación expost de los proyectos y programas de desarrollo, dando como resultado el diseño de una Metodología del Ciclo de Vida de Proyectos adaptada a las problemáticas del sector panelero colombiano descrita en forma general en la figura 1 : en su labor y les posibilite el desarrollo del sector con un resultado positivo para la región. A la vez, que se espera contar datos estadísticos que demuestren el beneficio de la incorporación de esta metodología en las actividades propias de la región. Por último, el principal y más importante trabajo, se encuentra representado en la ardua labor de divulgar la metodología con el fin de garantizar la adopción y usabilidad por los miembros de la comunidad panelera de Villeta.

Desde su fundación el 29 de septiembre de 1551, el municipio de Villeta (Cundinamarca) ha cultivado caña y producido panela. En la actualidad el municipio sigue teniendo como una de sus principales actividades la producción de panela, aunque en muchas fincas sigue siendo una actividad de tipo artesanal.

Hoy por hoy, este municipio sigue afectado por la falta de competitividad a pesar de los esfuerzos realizados por organismos gubernamentales y privados.

Figura $n^{\circ} 1$ :

Metodología propuesta del ciclo de vida del proyecto

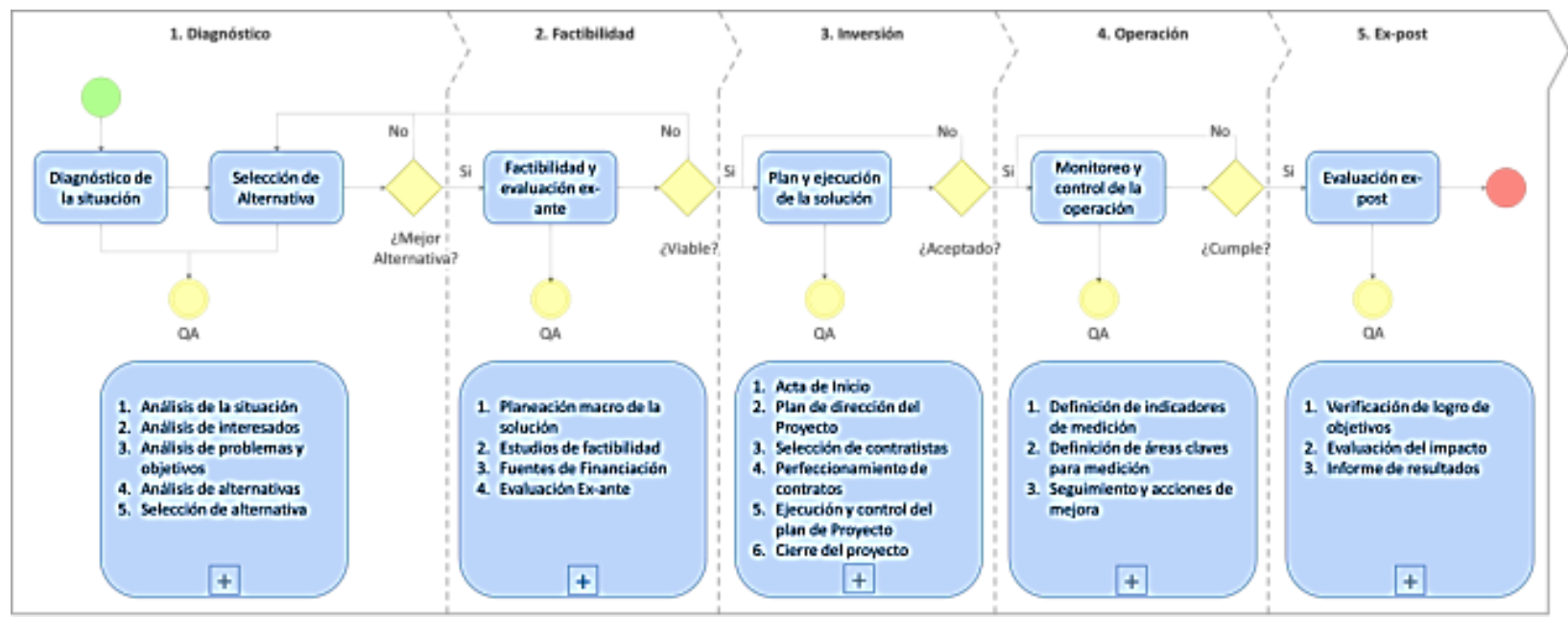

Fuente: Elaboración propia

Para el desarrollo de esta metodología se hizo especial énfasis en un desenvolvimiento práctico y fácil de su aplicación, que permita principalmente el involucramiento de la comunidad con un aporte significativo en la resolución de sus diferentes problemáticas a través de un trabajo participativo.

Cómo trabajo futuro, se espera implementar, a través de un trabajo de campo con el sector panelero de la región de Villeta-Cundinamarca, esta metodología proporcionándole a la comunidad todos los instrumentos y artefactos que permitan desde su perspectiva aunar esfuerzos para la resolución de las principales situaciones que se presenten
Factores como bajos ingresos, baja capacidad técnica para la producción, poca adaptación a nuevas tecnologías, dificultades en la comercialización del producto que no permite aumentar las ventas ni disminuir sus costos de distribución, migración de la mano de obra hacia la cabecera municipal y altos costos en la producción, han logrado una situación poco favorable para el desarrollo de la región. Por un lado los pequeños productores esperan pasivamente el apoyo de los organismos para cambiar su situación y por otro lado la administración no propone actividades que motiven a los productores a optimizar la productividad y fomentar el desarrollo de sus negocios. 
Meynsser Liliana Montero Acosta

Dentro del análisis de la situación actual del sector panelero y según lo expresa Ubaque en sus principales conclusiones acerca del fortalecimiento de la dinámica de adaptación a los cambios de los sistemas tradicionales de producción de panela en el municipio, en la Tabla 1 se extraen las propuestas estratégicas que principalmente se pueden ver beneficiadas con la aplicación del modelo metodológico propuesto:
En ese mismo orden de ideas, es importante precisar que la principal intención del modelo metodológico es suscitar la participación activa de la comunidad en la implementación y ejecución de alternativas de solución que propendan por mejorar sus condiciones.

\section{Tabla $\mathbf{N}^{\circ} 1$}

Propuestas estratégicas que se pueden ver beneficiadas con la aplicación del modelo metodológico

\begin{tabular}{|c|c|}
\hline Propuesta Estratégica & Actores Participantes \\
\hline $\begin{array}{l}\text { Promover el fortalecimiento o creación de formas asociativas de productores sobre la base inicial del ser } \\
\text { como orqanización y autogestores del desarrollo. }\end{array}$ & Alcaldia Municipal, SENA \\
\hline $\begin{array}{c}\text { Promover la creación de redes de cooperación local que permitan el trabajo conjunto en busca de } \\
\text { mejorar la capacidad de gestión, la competitividad, la comercialización y la definición de estrategias de } \\
\text { desarrollo. }\end{array}$ & $\begin{array}{l}\text { Alcaldia Municipal, } \\
\text { Productores, SENA, } \\
\text { Gobernación de } \\
\text { Cundinamarca, } \\
\text { FEDEPANELA }\end{array}$ \\
\hline $\begin{array}{l}\text { Promover diversificación de la economía rural, explorando no solo alternativas agrícolas y pecuarias sino } \\
\qquad \text { también considerando aquellos de origen no agricola. }\end{array}$ & $\begin{array}{l}\text { Alcaldia Municipal, } \\
\text { Gobernación de } \\
\text { Cundinamarca, SENA }\end{array}$ \\
\hline $\begin{array}{l}\text { Promover el consumo local de la panela como producto tradicional de origen rural y que identifica la } \\
\text { cultura e identidad panelera del municipio. }\end{array}$ & $\begin{array}{l}\text { Alcaldía Municipal, } \\
\text { Productores de panela }\end{array}$ \\
\hline $\begin{array}{l}\text { Realizar un proceso de formación para la creación de capacidades que mejoren y generen compromiso } \\
\text { de participación de la comunidad rural y productora de panela. }\end{array}$ & Alcaldía Municipal, SENA \\
\hline $\begin{array}{l}\text { Fortalecer y abrir espacio de participación local que permitan la discusión, análisis y resolución de las } \\
\text { problemáticas del territorio y en los que sea posible hacer la construcción y seguimiento de soluciones, } \\
\text { estrategias y decisiones relacionadas con la gestión y desarrollo del territorio rural. }\end{array}$ & Alcaldia Municipal \\
\hline $\begin{array}{l}\text { Implementar un programa de formación de jóvenes líderes y gestores rurales en el que se facilite el } \\
\text { desarrollo de habilidades y capacidades de participación y definición de actividades productivas y } \\
\text { empresariales encabezadas por jóvenes. }\end{array}$ & $\begin{array}{l}\text { Alcaldia Municipal, SENA, } \\
\text { Instituciones Educativas, } \\
\text { productores, FEDEPANELA }\end{array}$ \\
\hline $\begin{array}{l}\text { Crear escenarios de participación de la mujer habitante del sector rural que permitan dar un mayor } \\
\text { protagonismo a su contribución al desarrollo desde la vinculación de actividades productivas, } \\
\text { asociativas, y empresariales que lleven a la generación de confianza, empoderamiento y bienestar } \\
\text { socioeconómico de la mujer. }\end{array}$ & $\begin{array}{l}\text { Alcaldia Municipal, SENA, } \\
\text { Gobernación de } \\
\text { Cundinamarca, Mujer del } \\
\text { sector rural }\end{array}$ \\
\hline $\begin{array}{l}\text { Iniciar un proceso de revaloración de la identidad y cultura campesina panelera que estas contribuyan en } \\
\qquad \text { la construcción de tejido social, de gestión local y de adaptación a los cambios. }\end{array}$ & $\begin{array}{l}\text { Alcaldia Municipal, } \\
\text { Productores, FEDEPANELA }\end{array}$ \\
\hline $\begin{array}{l}\text { Fomentar la creación de grupos gestores ambientales que se conviertan en dinamizadores del } \\
\text { conocimiento ambiental, promotores de la recuperación y conservación de los recursos naturales, } \\
\text { movilizadores del interés de actuar frente a la prevención y solución de problemas ambientales } \\
\text { asociados al territorio. }\end{array}$ & Alcaldia Municipal, CAR \\
\hline $\begin{array}{c}\text { Fomentar la adopción de tecnologias agroforestales apropiadas para las pequeñas fincas y que tengan } \\
\text { la posibilidad de usos múltiples aportando a la diversificación de la finca y la protección de los recursos } \\
\text { naturales. }\end{array}$ & $\begin{array}{l}\text { Alcaldía Municipal, } \\
\text { Productores, CAR, } \\
\text { Gobernación de } \\
\text { Cundinamarca }\end{array}$ \\
\hline $\begin{array}{l}\text { Definir instrumentos de planificación como el Plan Operativo Anual bajo un enfoque participativo y de } \\
\text { vinculación de acciones enmarcadas en la dimensiones del desarrollo. }\end{array}$ & $\begin{array}{l}\text { Alcaldia Municipal, } \\
\text { Comunidad }\end{array}$ \\
\hline $\begin{array}{c}\text { Establecer alianzas interinstitucionales que permitan fortalecer la capacidad de respuestas de gestión e } \\
\text { inversión en el sector rural. }\end{array}$ & Alcaldía Municipal \\
\hline
\end{tabular}

Fuente: Ubaque González, Luz Lilia, op. cit., pp. 115-118 


\section{CONCLUSIONES:}

Los pequeños productores de panela de Villeta-Colombia se caracterizan por ser familias con poca capacidad económica, bajos niveles de educación técnica enfocada al trabajo productivo, desarraigo de la población joven, baja tecnificación de sus cultivos y producción de panela. Dichas características hacen que muchos de ellos no hayan logrado cumplir con las exigencias y normatividades gubernamentales para mantenerse en el mercado.

Con el fin de lograr la competitividad y el desarrollo de la región posibilitando la obtención de resultados económicos y sociales beneficiosos para los pequeños productores, para la región y para el país, es fundamental fomentar la participación activa de la comunidad y de esta manera explotar sus capacidades individuales que redunden en alternativas colectivas bien estructuradas para la consecución de recursos que les permita mejorar y desarrollar la producción y comercialización de la panela. Este proceso se puede realizar a través de la aplicación de mejores prácticas del ciclo de vida de proyectos.

Las mejores prácticas han demostrado, en el tiempo, que aportan significantemente en la obtención de mejores resultados para aquellas organizaciones, industrias y empresas que las adoptan. Las metodologías del ciclo de vida de proyectos no son ajenas a esta realidad que junto con la potencialización de la capacidad de las personas a través de ellas, seguramente lograrán una mejora significativa en la región de Villeta - Cundinamarca.

En este artículo se presenta una nueva metodología para la gestión del ciclo de vida de proyectos en pequeñas organizaciones comunitarias, a partir de su contenido se puede considerar, como línea futura de investigación, la aplicación del modelo en un grupo representativo de los pequeños productores de panela de Villeta Cundinamarca que permita evaluar si los resultados mejoran con su utilización. También es importante validar beneficios de su uso, como el trabajo en equipo, la integración, la sostenibilidad y la apropiación del conocimiento. Por otra parte y luego de su primera aplicación, puede resultar interesante ajustar el modelo y estandarizarlo para ser aplicado en muchos otros sectores productivos del país.

\section{REFERENCIAS:}

Acción Social, Manual de Formulación de Proyectos de Cooperación Internacional, Septiembre 2006 (disponible en http://www.accionsocial.gov.co)
AECl: Metodología de proyectos de cooperación para el desarrollo. AECl, Madrid, 1999

ALDUNATE, Eduardo, CÓRDOBA, Julio: Formulación de programas con la metodología de marco lógico. Manuales serie CEPAL - INSTITUTO LATINOAMERICANO Y DEL CARIBE DE PLANIFICACIÓN ECONÓMICA Y SOCIAL (ILPES). Abril 2011. $122 \mathrm{p}$.

BÁEZ, Juan, De TUDELA, Pérez, Investigación cualitativa, ESIC Editorail, 2007. 400p.

CAMACHO, Hugo. CÁMARA, Luis. CASCANTE, Rafael. SAINZ, Héctor El enfoque del marco lógico: 10 casos prácticos. Acciones de Desarrollo y Cooperación. Fundación CIDEAL. 2001. Recuperado de: http://www.preval.org/documentos/00423.pdf

CRAWFORD, Lynn: Global Body of Projects Management Knowledge and Standards. In The Wiley Guide to Managing Projects (eds P.W.G. Morris and J.K. Pinto), Jhon Wiley \& Sons, Inc., Hoboken, NJ, USA. Septiembre 2004. Chapter 46.

Delegatura de Protección de la Competencia. Estudios de Mercado Cadena productiva de la panela en Colombia: diagnóstico de libre competencia (2010-2012). Superintendencia de Industria y Comercio. Bogotá - Colombia.

GÓMEZ GALÁN, M., SAINZ OLLERO, H.: El ciclo del proyecto de cooperación al desarrollo. La aplicación del marco lógico. CIDEAL, Madrid, 1999.

GÓMEZ, Maluchi, EDISON, Javier: Manejo Agronómico de la Caña Panelera con Enfasis en el Control Biológico. Federación Nacional de Productores de Panela, FEDEPANELA. 2009.

GÓMEZ, Manuel. CÁMARA, Luis, Orientaciones para la aplicación del enfoque del marco lógico - Errores frecuentes y sugerencias para evitarlos. CIDEAL. 2003 (disponible en http://www.accionsocial.gov.co).

ILPES - INSTITUTO LATINOAMERICANO Y DEL CARIBE DE PLANIFICACIÓN ECONÓMICA Y SOCIAL Área de proyectos y programación de 
inversiones, Metodología del marco lógico para la planificación, el seguimiento y la evaluación de proyectos y programas, julio 2005.

ILPES - INSTITUTO LATINOAMERICANO Y DEL CARIBE DE PLANIFICACIÓN ECONÓMICA Y SOCIAL. Boletín del Instituto. Manuales serie CEPAL. Octubre 2004. 48 p.

KEMMIS, Stephen, MACTAGGART, Robín: Como planificar la Investigación-Acción. Laertes. Barcelona. 1988. 199 p.

LEWIN, Kurt, Action Research and Minority Problems. Journal of Social Issues, Volumen 2, Issue 4, pages 34-46. November 1946.

MONTERO ROJAS, Robinson: Formular y evaluar el proyecto de producción de panela en la vereda la Mazata del municipio de Villeta Cundinamarca. Corporación Universitaria Minuto de Dios Facultad de Ciencias Empresariales. Bogotá Colombia. 2008.

ORTEGÓN, Edgar, PACHECO, Juan Francisco, PRIETO, Adriana: Metodología del marco lógico para la planificación, el seguimiento y la evaluación de proyectos y programas. Manuales serie CEPAL. Julio 2005. 244 p.

ORTIZ, Alfredo, RIVERO, Guillermo: Desmitificando la Teoría del Cambio - Pact. Building Capacity Worldwibe. 2007.11 p.

OSORIO CADAVID, Guillermo: Buenas Prácticas Agrícolas -BPA- y Buenas Prácticas de Manufactura -BPMen la Producción de Caña y Panela. FAO, Gobernación de Antioquia, MANA, CORPOICA, Centro de Investigación "La Selva". Medellín Colombia. 2007. 202 p.

RODRÍGUEZ, G, GARCÍA, H, ROA DÍAZ, Z, SANTACOLOMA, P: Panela production as a strategy for diversifying incomes in rural area of Latin America. FAO, Rome (Italy). Agricultural Support Systems Div. 2007. $81 \mathrm{p}$.

UBAQUE GONZALEZ, Luz Lilia: Gestión en la Producción Panelera, Municipio de Villeta, Cundinamarca. Pontificia Universidad Javeriana - Facultad de Estudios Ambientales y Rurales, Maestría en Gestion Ambiental. Bogotá - Colombia. 2013. 